\title{
LA DIVERSIDAD EN LA EDUCACIÓN SUPERIOR: PROGRAMAS DE INTERVENCIÓN ACTUALES
}

\author{
Susana Tallón Rosales \\ Facultad de Ciencias de la Educación \\ Universidad de Granada \\ stallon@ugr.es \\ Carolina Fernández Jiménez \\ Facultad de Ciencias de la Educación \\ Universidad de Granada \\ Mirian Hervás Torres \\ Facultad de Ciencias de la Educación \\ Universidad de Granada \\ Marta Aparicio Puerta \\ Facultad de Ciencias de la Educación \\ Universidad de Granada \\ M. Tamara Polo Sánchez \\ Facultad de Ciencias de la Educación \\ Universidad de Granada
}

Fecha de Recepción: 24 Marzo 2019

Fecha de Admisión: 30 Abril 2019

\section{RESUMEN}

Una institución formativa más eficaz y una educación más humana se basa en, atender a los alumnos independientemente si tienen discapacidad, pertenecen a una cultura, raza o religión diferente (Arnaiz Sánchez, 1996). Partiendo de esta premisa, en la Educación Superior el planteamiento es común, puesto que es la etapa educativa preparatoria para la inserción al mercado laboral. Hablamos pues, de una educación inclusiva que abarca una actitud, un sistema de valores y un conjunto de acciones, formando un proceso de innovación (Moreno Tallón, 2010). Es decir, compensar las desigualdades en colectivos desfavorecidos que pudieran presentar dificultades de aprendizaje y emanen del tal desventaja y tratamiento social-escolar.

Este trabajo pretende mostrar un recorrido práctico de diferentes intervenciones en el ámbito de la Educación Superior, ofreciendo una perspectiva actual de cómo se trabaja la diversidad en las universidades. Para ello, se ha realizado una revisión de la literatura en diferentes bases de datos, para conocer las aportaciones de distintos programas que han intervenido en el contexto de la formación universitaria en la respuesta a la diversidad de su alumnado. Este conocimiento ayudará a mejorar la respuesta a la atención a la diversidad, y promover sinergias entre instituciones y profesionales en pro de generar más propuestas de actuación en contextos universitarios. 


\section{LA DIVERSIDAD EN LA EDUCACIÓN SUPERIOR: PROGRAMAS DE INTERVENCIÓN ACTUALES}

Palabras clave: diversidad; educación superior; programas de intervención; formación

\section{ABSTRACT}

Diversity in higher education: current intervention programs. An educational institution more effective and more humane education is based on, serve students regardless if they have disabilities, belong to a culture, race or religion other, (Arnáiz Sánchez, 1996). Starting from this premise, in higher education approach is common, since it is the preparatory educational stage for the inclusion in the labour market. We speak, of an inclusive education which encompasses an attitude, a value system and a set of actions, forming a process of innovation, (Moreno Tallon, 2010). Therefore to compensate the inequalities in collective underprivileged or disadvantaged social, economic, cultural, geographical, ethnic or otherwise could have learning difficulties which stem from the such disadvantage and social-escolar treatment.

In this sense, this work aims to show a practical tour of different interventions in the field of higher education, offering a current perspective of how to work the diversity in the universities. It has conducted a review of the literature on different databases, to learn about the contributions of different programs that have intervened in the context of university education in response to the diversity of its student body. This helps improve the response to the attention to diversity and its implementation in the classroom, as well as to promote synergies between institutions and professionals in favor of generating further proposals for action in University contexts.

Keywords: diversity; higher education; intervention programs; training

\section{INTRODUCCIÓN}

En los últimos años, la educación ha adquirido una revalorización contextual, convirtiéndose ésta en el instrumento que ofrece la sociedad para compensar desigualdades de cualquier índole. Sin especificar etapas educativas, respecto a la obligatoriedad o no de las mismas y sus características específicas de formación según los colectivos, la enseñanza universitaria puede ser una posible etapa educativa más en la formación global del estudiante, adquiriendo ésta una mayor importancia en la atención al alumnado con Diversidad y/o discapacidad en particular. En el ámbito del Espacio Europeo de Educación Superior en la Declaración de La Sorbona (1998), se promueve una dimensión de la Educación Superior, caracterizada por un desarrollo curricular más eficaz, cooperación institucional, así como programas integrados de estudios, de formación y de investigación. El protagonista de este proceso es el propio aprendizaje a lo largo de toda la vida, como elemento esencial para alcanzar, entre otros aspectos, la mejora de la cohesión social, la igualdad de oportunidades y la calidad de vida (MECD, 2003).

Respecto al marco legislativo en la educación superior, es poco lo que se encuentra relativo a esta etapa de último nivel, en relación con las otras etapas educativas. En concreto se detalla en el anexo y en la disposición adicional vigésima cuarta, "de la inclusión de las personas con discapacidad en las universidades" (Resolución del Consejo de 5 de mayo 2003, sobre la igualdad de oportunidades en educación y formación para los alumnos y estudiantes con discapacidad), destaca: 1. Las Universidades garantizarán la igualdad de oportunidades de todos los miembros de la comunidad universitaria con discapacidad. 2. Los estudiantes y los demás miembros con discapacidad de la comunidad universitaria no podrán ser discriminados por razón de su discapacidad, entre otros aspectos relevantes.

La diversidad es algo inherente a cualquier ser vivo que habite en la tierra. En ningún lugar del mundo podremos encontrar dos personas iguales ya que todos hemos adquirido "esa diversidad" que es lo que nos hace ser diferentes, únicos e irrepetibles. En este proceso de atención a la Diversidad, son múltiples los términos implicados, tales como, los conceptos de sociedad, cultura, 
educación, interrelacionados entre sí. Esta terminología permite analizar el fenómeno de la exclusión de la educación, las posibilidades que ofrece la escuela inclusiva en la atención a la Diversidad, la cual potencia desarrollar y fomentar actitudes positivas de valoración y respeto. La escuela, centros educativos y la propia universidad, constituyen instituciones que gestionan colectivos más que individuos, la sociedad espera unos comportamientos, competencias y perfiles determinados. Esto no debe entenderse en el sentido de que la escuela estimule la actividad colectiva espontánea o autoorganizada, pues lo que hace es justamente lo contrario (Fernández, 1997). Dicho aprendizaje de roles va intrínseco a la educación, por su relación a la cultura generada por dichos colectivos que normatizan a las sociedades actuales.

Al abordar la complejidad de la Diversidad debida a factores socioeducativos y culturales, es necesario analizar diversos fenómenos relacionados con los conceptos básicos de discapacidad, desigualdad y desventaja en educación. Factores que pueden llevar a la exclusión social de los jóvenes desde edades tempranas debido a experiencias escolares no acordes, además de otras capacidades implicadas, tales como la motivación de logro, habilidades sociales y resolución de conflictos. En definitiva, existe en términos generales, una homogeneización en y del proceso de enseñanza-aprendizaje, en cualquier etapa educativa, pero es prioritario fomentar y propiciar una heterogeneidad en la educación y aún con mayor énfasis en la educación superior, por constituir la formación que cierra dicho proceso educativo y que es preparatoria para la inserción laboral.

Apostar por una enseñanza y una escuela en donde destaca el valor de lo diverso, así como la diferencia en sí; es una apuesta por una educación enriquecedora ya que destaca lo positivo y lo suma, en lugar de considerarlo como algo negativo y discriminatorio. Potenciar y aceptar que lo diverso y diferente es algo normal y enriquecedor, pero no solamente en la escuela y el centro educativo, sino también que la sociedad debe participar de esta actitud y lograr un sentimiento positivo acerca de los derechos de los alumnos con necesidades educativas especiales (Olson, 2000). Es destacable la evolución en torno a conceptos relacionados con el ámbito de la Diversidad, que a día de hoy, se consideran peyorativos o poco apropiados, términos como "deficiente", "subnormal", "disminuido", centrados en la deficiencia del sujeto y/o en la subnormalidad. Sin tener en cuenta variables externas a la persona, tales como circunstancias sociales, económicas, familiares, culturales. A finales del pasado siglo, ha acontecido en todos los países, una reestructuración en la legislación educativa a favor de la Integración, eludiendo aquellas corrientes teóricas meramente de origen médico, para enfatizar todo lo educativo como intervención y atención hacia las personas con discapacidad (Agencia Europea para el Desarrollo de la Educación Especial, 1998; Hegarty, 1996).

Este reconceptualización de términos define los cambios legislativos y sobre todo actitudinales acaecidos desde décadas atrás hasta el momento, en pro de la mejora en la atención a la Diversidad en la educación. En la actualidad, el concepto de inclusividad, recoge esta apertura actitudinal y procedimental, atendiendo a la persona que presenta diferencias de forma individual. Dichos contextos inclusivos, evidencian que los alumnos con discapacidades aprenden de una forma más eficaz y ajustada (Bunch, 2008).

En la era de la inclusividad surgen términos como "escuela comprensiva", "escuelas eficaces", "escuelas inclusivas", "inclusión total", "educación inclusiva", "integración total”, "unificación de sistemas", para definir a los actuales sistemas educativos, como más eficaces al reconstruir el concepto de educación incluyendo su variante más humana para atender al alumnado con independencia de tener 0 no discapacidades, o pertenecer a una cultura, raza o religión diferente (Arnaiz Sánchez, 1996; León Guerrero, 1998). Atender a la Diversidad en los procesos educativos requiere de modalidades de actuación diferentes para grupos en progresiva diferenciación, tales como el refuerzo educativo, adaptaciones y diversificaciones. Así como actuaciones específicas en el aula, 


\section{LA DIVERSIDAD EN LA EDUCACIÓN SUPERIOR: PROGRAMAS DE INTERVENCIÓN ACTUALES}

flexibilidad de agrupamientos, trabajo cooperativo y autónomo. En concreto, la respuesta a la Diversidad se ha de plasmar en la adaptabilidad y flexibilidad del currículum (Torres González, 1999).

Por tanto, hay que atender a la Diversidad desde el principio de flexibilidad, adaptación y apertura de la educación, surgiendo así una nueva visión de la enseñanza y de la escuela (Cano González 2003). Y para ello, hay que destacar el concepto de inclusión como un todo. Parrilla (2002) establece que "la inclusión es sobre todo un fenómeno social", es decir, la inclusión es un proceso procedente de las sociedades. La inserción social y laboral constituye un aspecto importante para las personas que presentan algún riesgo de exclusión ya que por sus determinadas situaciones les es más difícil tener acceso a un trabajo, por tanto aquellas personas que decidan formarse en la Universidad, ésta será el puente para la conciliación entre su formación y su puesto de trabajo. Es por este sentido, que la gestión de la Diversidad en el tramo universitario, no debe centrarse sólo en el acceso sino en potenciar esas diferencias, y así formarlos como personas únicas y capaces de obtener la inserción en el mercado laboral, función ésta perteneciente a la Educación Superior, entre otras.

\section{OBJETIVOS}

El objetivo de este trabajo es realizar una revisión sobre actuaciones en el ámbito universitario, en pro de la atención a la Diversidad en el alumnado que generan medidas beneficiosas y ofreciendo una educación integral y compensatoria, con lo cual aporta aspectos necesarios a tener en cuenta como futuras medidas en la Educación Superior.

\section{DESARROLLO DE LA PRÁCTICA/EXPERIENCIA}

Para llevar a cabo el objetivo propuesto, se ha llevado a cabo una revisión de la literatura entre Ios años 2008 y 2018. Se realizó una búsqueda en las bases de datos: Web of Science, Proquest, Proques, Scopus y Dialnet. En cuanto a los descriptores, se incluyeron las diferentes palabras clave: "programas universitarios alumnos con discapacidad», "programas de atención la diversidad en la universidad», "diversidad», "educación superior», "formación», "discapacidad», "university programs students with diversity», "diversity care programs at the university», "diversity», "higher education», "training», "disability» entre las más importantes.

De un total de 36 artículos encontrados, se descartaron 28 de ellos. En total se seleccionaron 8 artículos que han realizado estudios y/o programas con el colectivo de alumnado universitario con discapacidad, así como los propios servicios que ofrecen diversas universidades españolas. La selección de las publicaciones versa en torno a los siguientes criterios: (1) programas implementados con alumnos discapacitados universitarios, (2) resultados de los servicios inherentes en las universidades hacia la diversidad y (3) recursos propuestos en la universidades españolas para el alumnado y profesorado en torno a la discapacidad.

\section{EVIDENCIAS}

A continuación, se presenta un resumen con los resultados encontrados (Tabla 1), identificando sus autores y año de publicación, colectivo al que va dirigido, y tipo de programa y/o estudio realizado. 
Tabla 1

Resumen de las actuaciones dirigidas a la Atención a la Diversidad en alumnos universitarios

\begin{tabular}{|c|c|c|}
\hline Autores y año & Colectivo & Tipo de programa \\
\hline $\begin{array}{l}\text { Diego Jesús } \\
\text { Luque Parra y } \\
\text { Gemma } \\
\text { Rodríguez } \\
\text { Infante (2008) }\end{array}$ & $\begin{array}{l}\text { De } 232 \text { alumnos } \\
\text { universitarios, } 71 \\
\text { solicitan este } \\
\text { servicio. } \\
\text { Universidad de } \\
\text { Málaga }\end{array}$ & $\begin{array}{l}\text { Servicio de apoyo al alumnado con } \\
\text { discapacidad de la Universidad de } \\
\text { Málaga. Intervención } \\
\text { psicopedagógica. } \\
\text { Cuenta con diferentes apoyos: } \\
\text { alumno colaborador, cuidadores } \\
\text { ayudas económicas transporte y } \\
\text { préstamo de ayudas técnicas }\end{array}$ \\
\hline $\begin{array}{c}\text { María José Fiuza } \\
\text { Asorey y Silvia } \\
\text { Sierra Martínez } \\
\quad(2017)\end{array}$ & $\begin{array}{l}88 \text { estudiantes de } \\
\text { la Facultad de } \\
\text { Formación del } \\
\text { Profesorado. } \\
\text { Universidad de } \\
\text { Santiago de } \\
\text { Compostela }\end{array}$ & $\begin{array}{l}\text { Diseño cuentos inclusivos en } \\
\text { colaboración con la Organización } \\
\text { Nacional de Ciegos Españoles } \\
\text { (ONCE). El proceso pretende } \\
\text { iniciarles en el Aprendizaje- } \\
\text { Servicio }\end{array}$ \\
\hline $\begin{array}{l}\mathrm{M}^{\mathrm{a}} \text { Tamara Polo } \\
\text { Sánchez y M }{ }^{\mathrm{a}} \\
\text { Dolores López } \\
\text { Justicia (2011) }\end{array}$ & $\begin{array}{c}25 \text { estudiantes } \\
\text { universitarios } \\
\text { discapacitados. } \\
\text { Universidad de } \\
\text { Granada }\end{array}$ & $\begin{array}{c}\text { Informar y capacitar sobre técnicas } \\
\text { de búsqueda de empleo a un grupo } \\
\text { de estudiantes universitarios con } \\
\text { discapacidad física y/o sensorial } \\
\text { utilizando nuevas tecnologías de } \\
\text { acceso a la información }\end{array}$ \\
\hline $\begin{array}{l}\text { Eva María Nava- } \\
\text { Caballero (2012) }\end{array}$ & $\begin{array}{l}9 \text { alumnos } \\
\text { universitarios con } \\
\text { discapacidad, } \\
\text { Universidad de } \\
\text { León }\end{array}$ & $\begin{array}{c}\text { Se llevó a cabo un estudio de } \\
\text { casos, que ofrece datos de la } \\
\text { situación del } 12,7 \% \text { de la población } \\
\text { objeto de estudio }\end{array}$ \\
\hline $\begin{array}{l}\text { María Ángeles } \\
\text { Segura García, } \\
\text { José Manuel } \\
\text { Martínez Vicente } \\
\text { y Isabel García } \\
\text { Martínez (2015) }\end{array}$ & $\begin{array}{c}30 \text { titulados } \\
\text { universitarios } \\
\text { Universidad de } \\
\text { Almería }\end{array}$ & $\begin{array}{l}\text { Se le aplicó un cuestionario de } \\
\text { biodatos universitarios y un } \\
\text { inventario de factores de elección } \\
\text { vocacional. Se empleó un diseño } \\
\text { correlacional e inferencial }\end{array}$ \\
\hline $\begin{array}{l}\text { Alejandro } \\
\text { Rodríguez } \\
\text { Martín, Emilio } \\
\text { Álvarez Arregui } \\
\text { y Rosa García- } \\
\text { Ruiz (2014) }\end{array}$ & $\begin{array}{c}85 \text { Profesores } \\
\text { universitarios y } \\
1015 \text { alumnos } \\
\text { universitarios } \\
\text { Universidad de } \\
\text { Cantabria y de } \\
\text { Oviedo }\end{array}$ & $\begin{array}{l}\text { Los resultados obtenidos revelan } \\
\text { una actitud positiva hacia la } \\
\text { inclusión universitaria de los } \\
\text { estudiantes con discapacidad pero } \\
\text { una opinión negativa del } \\
\text { profesorado hacia las adaptaciones } \\
\text { curriculares }\end{array}$ \\
\hline $\begin{array}{c}\text { María José } \\
\text { Vieira Aller y } \\
\text { Camino Ferreira } \\
\text { Villa (2011) } \\
\end{array}$ & $\begin{array}{l}\text { Universidades } \\
\text { Comunidad } \\
\text { Autónoma de } \\
\text { Castilla y León }\end{array}$ & $\begin{array}{l}\text { A través del estudio de casos, se } \\
\text { analiza el funcionamiento de los } \\
\text { servicios apoyo a la discapacidad }\end{array}$ \\
\hline $\begin{array}{c}\text { Raquel Suriá } \\
\text { Martínez, Tomás } \\
\text { Ordoñez Ruíz y } \\
\text { Domingo } \\
\text { Martínez Maciá } \\
\text { (2011) }\end{array}$ & $\begin{array}{c}2643 \text { alumnos } \\
\text { universitarios } \\
\text { (1998 y el 2008) } \\
\text { Universidad de } \\
\text { Alicante }\end{array}$ & $\begin{array}{c}\text { Este estudio analiza la evolución } \\
\text { de las demandas de apoyo } \\
\text { psicoeducativo, en relación al } \\
\text { género, titulación y curso } \\
\text { académico }\end{array}$ \\
\hline
\end{tabular}




\section{LA DIVERSIDAD EN LA EDUCACIÓN SUPERIOR: PROGRAMAS DE INTERVENCIÓN ACTUALES}

Tras la revisión efectuada, a través de las diversas búsquedas en las base de datos mencionadas, los resultados hallados muestran, a priori, que si se realizan programas y actividades, pero falta profundizar en el nivel de ejecución y seguimiento, limitándose en la gran mayoría a constituir un servicio de atención o solicitar cita en el mismo.

En este sentido, el estudio de Luque Parra y Rodríguez Infante (2008), sobre el uso y disponibilidad del servicio de apoyo a alumnado con discapacidad de la Universidad de Málaga, en concreto de la intervención psicopedagógica, ofrece como resultado, de los 71 alumnos solicitantes, se mantuvieron 36 entrevistas de asesoramiento, de éstos, 34 recibieron un tratamiento más personalizado y, en doce casos, se ha asesorado a los padres sobre los recursos que ofrece la Universidad, así como al profesorado en torno a las adaptaciones curriculares necesarias.

Con iniciativas, como la de los autores, Fiuza Asorey, y Sierra Martínez (2017), a través de la realización de cuentos inclusivos, ha permitido al alumnado universitario una mayor reflexión sobre la discapacidad en general y la discapacidad visual en particular, desde la mirada de la escuela inclusiva. En este estudio, han participado un total de 88 alumnos matriculados en la asignatura de Dificultades de aprendizaje y trastornos del desarrollo del Grado de Educación Primaria, dos maestros itinerantes especialistas en discapacidad visual; $y$, por último, se eligieron al azar tres niñas con visión normal), tres con restos visuales y tres con ceguera escolarizados en diferentes centros educativos de dicha provincia.

Otros estudios en este ámbito de la Diversidad en la universidad, empiezan a destacar la importancia no sólo de su acceso y tratamiento, sino una vez finalizada la formación universitaria, para obtener un puesto de trabajo. Destacamos, a las autoras Polo Sánchez y López Justicia (2011), y su programa de formación para potenciar en ellos la adquisición de conocimientos y habilidades necesarias para buscar empleo. Los resultados de la valoración del programa, indican el gran interés y utilidad para los participantes por recibir mayor información y asesoramiento en estrategias y técnicas de búsqueda de empleo, y formación en nuevas tecnologías y cursos de especialización. Las discapacidades de los alumnos participantes eran 5 con discapacidad visual, 12 con discapacidad auditiva y 8 con discapacidad motora.

Eva María Nava-Caballero (2012), en su investigación, de naturaleza cualitativa, llevó a cabo un estudio de caso, con una muestra 9 alumnos con discapacidad, de los 71 que estaban matriculados en la Universidad de León. Han participado dos alumnos con deficiencia visual, una con deficiencia auditiva, una con discapacidad psíquica y cinco con discapacidad física. En general un 66,6\%, se encuentra integrado a pesar de haber percibido algún tipo de discriminación dentro de la comunidad universitaria y el 33,4\% restante no está integrado; y son alumnos con discapacidad desde su nacimiento 0 en los primeros años de sus vidas. Demandan personas para que les ayuden, y exigen más información sobre las discapacidades para toda la comunidad universitaria.

María Ángeles Segura García et al (2015), en la Universidad de Almería, emplearon un diseño correlacional e inferencial realizándose análisis de correlaciones, de regresión y la pruebas de Krusal-Wallis y $U$ de Mann-Whitne en los análisis inferenciales. Los resultados obtenidos destacaron que, el desarrollo vocacional eficaz quedó asociado, a los gustos y preferencias en las elecciones que realizaron. Y a mayor desarrollo vocacional eficaz, menos influencia ejerció los factores de carácter sociogénico y su propia discapacidad en la elección vocacional.

En este contextos de estudios respecto a la diversidad en la universidad, los autores, Rodríguez Martín et al (2014), priorizan la voz del profesorado, como protagonistas en este proceso, ya que el actual modelo universitario requiere del profesorado nuevas competencias para atender a las personas con discapacidad. Como resultado de este programa, el profesorado afirma que los estudiantes con discapacidad a los que han impartido docencia, acuden con menos frecuencia que el 
resto de estudiantes a sus clases $(64.8 \%$ ) y a las tutorías (59.3\%). Con respecto al rendimiento académico de los estudiantes con discapacidad, el $46.3 \%$ del profesorado opina que es semejante al del resto de estudiantes y el $40.7 \%$ que es inferior.

María José Vieira Aller y Camino Ferreira Villa (2011), analizan de forma descriptiva la evolución de las demandas de apoyo psicológico y psicoeducativo que los alumnos solicitan al servicio de asesoramiento psicológico de la universidad de Alicante. Se examina el tipo de apoyo solicitado y su relación con el género, titulación y curso académico de los estudiantes. La metodología seguida se basa en el estudio de casos, siendo las unidades de análisis los servicios de atención a la discapacidad de las universidades de Comunidad Autónoma de Castilla y León. El cuestionario fue enviado a las ocho universidades (4 públicas y 4 privadas). Dando como resultado una necesaria estructuración y coordinación de estos servicios.

De los últimos estudios y/o programas destacados, el realizado por Suriá Martínez, Ordoñez Ruíz y Martínez (2011), destaca la importancia del apoyo psicológico demandado por estudiantes universitarios con algún tipo de discapacidad en general, existe una mayor frecuencia en la solicitud de asesoramiento por parte de las mujeres que de los varones, así como los estudiantes de Magisterio, Biología, Derecho y Arquitectura acuden más a demandar apoyo. De los 2643 participantes, muestran un incremento en demandas de atención no sólo académicas sino de atención psicológica.

\section{CONCLUSIONES}

De este discurso parte, promover actuaciones que favorezcan la atención a la Diversidad en alumnado universitario de las personas que puedan necesitar una adaptación, apoyo, y/o recurso específico que refuerce el sentido estratégico compartido para abordar su situación, siendo su eje principal el nivel y tipo de apoyos que reciben estos colectivos.

En la indagación de literatura sobre esta temática, es cierto que existen iniciativas al respecto, pero en su mayoría consiste en estudios sobre opinión y valoración de servicios de acogida por parte de las universidades hacia los alumnos con discapacidad. Un dato resultante, es la limitación es estudios que constituyan programas específicos con actividades y pautas adaptadas a la mejora y bienestar de dicho colectivo universitario. En esta investigación, se ha visto mermada la disponibilidad en bases de datos y hemerotecas, de informes, publicaciones y artículos que aborden la puesta en acción de un bagaje de programas al respecto, y sus resultados. Además denotamos una carencia de investigaciones en relación a qué necesidades demandan los alumnos universitarios discapacitados, sobre la propia entidad y la salida de la misma hacia el mercado de trabajo. Esto se traduce en que las entidades universitarias si están concienciadas hacia la Diversidad en el alumnado, pero falta un bagaje de programas y actuaciones adaptadas a las necesidades de dichos alumnos y sus diversas discapacidades, como parte esencial de la cartera de servicios propia que ofrece cada universidad en el territorio español.

Este resultado nos lleva a proponer a la comunidad académica, nuevas líneas de investigación en pro de diseñar medidas específicas de actuación para el colectivo de alumnos universitarios que puedan presentar discapacidad en cualquiera de sus variantes, no como un servicio de atención psicoeducativa, que es el resultado mayoritario existente en nuestras universidades, sino en forma de actuaciones más pragmáticas que permitan la mejora no sólo personal, sino una acorde preparación para la pronta vida laboral. En este sentido las futuras líneas de investigación serían las siguientes:

1. Estudio comparativo de las necesidades académicas, y de adaptación del alumnado universitario con discapacidad, en las distintas comunidades autónomas.

2. Aplicación de un programa de orientación y acompañamiento en la inserción para alumnos universitarios con discapacidad. 


\section{LA DIVERSIDAD EN LA EDUCACIÓN SUPERIOR: PROGRAMAS DE INTERVENCIÓN ACTUALES}

3. Diseño de actividades propias en las universidades, como medidas de atención y seguimiento de las diversas discapacidades que presenta el alumnado universitario.

4. Formación al profesorado universitario sobre la atención y acogida en las aulas de los alumnos que presentan discapacidad.

Esta línea de investigaciones está acorde a otros estudios realizados que muestran similitud en los resultados ofrecidos, destacando la falta de propuestas más prácticas en las instituciones universitarias respecto a la Diversidad. Dichos estudios ponen de manifiesto y priorizan la importancia no sólo de las percepciones que desde el ámbito universitario existe hacia los alumnos discapacitados, como parte inicial para acometer dicha temática y promover en los organismos e instituciones una colaboración al respecto; la Diversidad por sus múltiples diferencias necesita abordarla desde todas las posibles entidades que tengan labor en dicho cometido y así impulsar programas de formación y promover mejoras en la actividad docente universitaria, favoreciendo una intercolaboración entre los organismos implicados en la mejora de las distintas discapacidades existentes. Así se impulsará la función y rol del profesional docente universitario, proporcionándole recursos que favorezcan la inclusión de los estudiantes discapacitados presentes en las universidades. (Luque Parra y Luque Rojas, 2010; Suriá Martínez, 2012).

\section{REFERENCIAS BIBLIOGRÁFICAS}

Agencia Europea para el Desarrollo de la Educación Especial (1998). Integración en Europa: Provisión para alumnos con necesidades educativas especiales. Tendencias en 14 países. Dinamarca: Comisión Europea. Recuperado de http://www.european agency.org

Arnaiz Sánchez, P. (1996). Las escuelas son para todos. Siglo Cero, 27(2), 25-34.

Bunch, G. (2008). Claves Para una educación inclusiva exitosa. Una mirada desde la experiencia práctica. Revista Internacional de Educación Inclusiva, 1,1, 18-30.

Cano González, R. (Coord.) (2003). Bases pedagógicas de la educación especial. Manual para la formación inicial del profesorado. Madrid: Biblioteca Nueva.

Fernández, M. (1997). La escuela a examen. Madrid: Pirámide.

Fiuza Asorey, y Sierra Martínez (2017). Creando y adaptando materiales para compartir: una experiencia con futuros maestros. Educatio Siglo XXI, 35(3), 153-174. http://dx.doi.org/10.6018/j/308951

Hegarty, S. (1996). La Educación Especial en Europa. Revista Española de Pedagogía, 204. 345360. León Guerrero, M. J. (1998). De la integración escolar a la escuela inclusiva 0 escuela para todos. En M. A. Lou Royo y N. López Urquízar (Coords.), Bases psicopedagógicas de la educación especial (pp. 39-61). Madrid: Pirámide.

Luque Parra, D. J. y Luque-Rojas, M. J. (2010). Acción tutorial y discapacidad: sugerencias de trabajo en el aula. Revista Española de Orientación y Psicopedagogía, 21(3), 633-642

MECD (2003). La Integración del Sistema Universitario Español en el Espacio Europeo de Enseñanza Superior (Documento-Marco). Madrid: Ministerio de Educación Ciencia y Deporte.

Nava-Caballero, E. (2012). El acceso y la integración de los estudiantes con discapacidad en la universidad de León. Revista Complutense De Educación, 23(2), 293-316.

Olson, L. P. (2000). He ain't heavy, he's my brother. Exceptional siblings: resiliency and vulnerability factors explored in adults with disabled siblings. Dissertation Abstracts International Section A: Humanities \& Social Sciences, 60(7-A), 24-46.

Parrilla, A. (2002). Acerca del origen y sentido de la educación inclusiva. Revista de Educación, 327, 11-29.

Resolución del Consejo de 5 de mayo de 2003, sobre la igualdad de oportunidades en educación y 
formación para los alumnos y estudiantes con discapacidad. Diario Oficial de la Unión Europea. DOC 134/04 (6-7). Disposición adicional vigésima cuarta. De la inclusión de las personas con discapacidad en las universidades.

Rodríguez Martín, A., Álvarez Arregui, E., y García-Ruiz, R. (2014). La atención a la diversidad en la universidad: el valor de las actitudes. Revista Española De Orientación y Psicopedagogía 25(1), $44-61$.

Sánchez, M., Polo, T., y Justicia, M. D. L. (2011). Transición al mundo laboral de estudiantes universitarios con discapacidad: experiencia de un programa formativo. Revista Española De Orientación y Psicopedagogía 22(3), 302-313.

Segura García, M.A. Martínez Vicente, J. M. y García Martínez, I. (2015). Relación entre el desarrollo vocacional eficaz y los factores de elección. Revista Española De Orientación y Psicopedagogía, 26(3), 8-25.

Suriá Martínez, R., Ordoñez Ruíz, T., y Martínez Maciá, D. (2011). Estudio descriptivo de la evolución del servicio de asesoramiento psicológico y psicoeducativo de la universidad de alicante. Revista Española De Orientación y Psicopedagogía, 22(2), 151-164.

Suriá Martínez, R. (2012). Discapacidad e integración educativa: ¿Qué opina el profesorado sobre la inclusión de estudiantes con discapacidad en sus clases? Revista Española de Orientación y Psicopedagogía, 23(3), 96-109.

Torres González, J. A. (2009). Desde la integración escolar a la educación inclusiva: nuevas realidades, nuevas necesidades. En P. Gútiez (Coord.), Tendencias actuales en la atención a las necesidades educativas especiales (pp. 13-34). Fundación UIMP: Grupo Editorial Universitario. 
